\title{
Hyers-Ulam Stability and Existence Criteria for the Solution of Second-Order Fuzzy Differential Equations
}

\author{
Noor Jamal, ${ }^{1}$ Muhammad Sarwar ${ }^{10},{ }^{1}$ and M. Motawi Khashan ${ }^{2}$ \\ ${ }^{1}$ Department of Mathematics, University of Malakand, Chakdara Dir (L), Pakistan \\ ${ }^{2}$ Department of Basic Sciences, Common First Year, King Saud University, Riyadh 11451, Saudi Arabia \\ Correspondence should be addressed to Muhammad Sarwar; sarwarswati@gmail.com
}

Received 11 December 2020; Revised 31 December 2020; Accepted 29 March 2021; Published 28 May 2021

Academic Editor: Zoran Mitrovic

Copyright (c) 2021 Noor Jamal et al. This is an open access article distributed under the Creative Commons Attribution License, which permits unrestricted use, distribution, and reproduction in any medium, provided the original work is properly cited.

In this paper, existence, uniqueness, and Hyers-Ulam stability for the solution of second-order fuzzy differential equations (FDEs) are studied. To deal a physical model, it is required to insure whether unique solution of the model exists. The natural transform has the speciality to converge to both Laplace and Sumudu transforms only by changing the variables. Therefore, this method plays the rule of checker on the Laplace and Sumudu transforms. We use natural transform to obtain the solution of the proposed FDEs. As applications of the established results, some nontrivial examples are provided to show the authenticity of the presented work.

\section{Introduction}

Zadeh [1] introduced the concept of fuzziness in the set theory. The complexity of uncertainty as ambiguity in a real-life scenario is dealt properly with fuzzy set theory. The mathematical tool of fuzzy set theory deals with uncertainty in the real-life problem in a better way. The fuzzy set theory has suggestions for nonclassical and higher order fuzzy sets for different specialized purposes. In this direction, Chang and Zadeh used fuzzy sets and initiate the concept of fuzzy mapping and control [2]. The work of many researchers on fuzzy mappings and control puts the foundation of elementary fuzzy calculus. For detail, see [3-7]. For the last two decades, the valuable interest of fuzzy integral and differential equations has extended classical calculus to modern fuzzy calculus. Fuzzy differential and integral equations are the well-equipped mathematical tools to deal properly with physical models in the fuzzy environments. The solutions of every fuzzy differential and integral equation do not exist. Therefore, some strategy is required to insure whether the solution of FDEs exists or not. The existence theory is one of the best research areas in the field of fuzzy differential equations (FDEs). Before dealing a physical model, it is important to know whether its solution exists. Now, if unique solution of a physical model exists, then a physical model is dealt properly. The existence of a unique solution of fuzzy differential equation properties of differentiable fuzzy mappings was studied by Kaleva [5]. Liu and Liu [8] introduced self-duality credibility measure for the measurement of fuzzy event. Moreover, they studied the existence of unique solutions of FDEs. The existence and uniqueness result for FDEs with linear growth and Lipschitz conditions was discussed by Fei et al. [9]. The uniqueness result for the FDEs with nonLipschitz coefficients was investigated by Chen and Qin [10] for more detail (see $[6,11,12]$ ).

Stability analysis of differential equations (DEs) is another most important and remarkable area in the qualitative theory. The stability of DEs has been studied by various researchers with different concepts like Lyapunov stability, asymptotic stability, and Ulam stability. The first effort of Ulam stability was initiated by Ulam [13], and just after one year, Hyers [14] studied the stability of the linear functional equation known as Hyers-Ulam stability. Oblaza [15] proved the Hyers-Ulam stability of linear DEs; for further detail of Hyers-Ulam, see [16-19]. Shen [20] investigated the Ulam stability of first-order linear FDEs.

The Laplace and Sumudu transforms are commonly used for the solutions of differential equations. The natural transform introduced by Khan and Khan [21] has speciality to converge to both Laplace and Sumudu transforms only by 
changing the variables. Therefore, the natural transform method plays the rule of checker on the Laplace and Sumudu transforms. The applications of the natural transform method turn out to be well for solutions of differential equations, see [21-23].

The aim of this work is to study the existence, uniqueness, and Hyers-Ulam stability of second-order FDEs. For this purpose, the corresponding second-order FDEs are reduced to equivalent systems of fuzzy integral equations. Using the concept of Hukuhara generalized differentiability, existence, uniqueness, and Hyers-Ulam stability of the equivalent system of integral equations are discussed. We use the natural transform method to solve second-order FDEs. The last Hyers-Ulam stability of the numerical problem is discussed. Two nontrivial examples are given to show the authenticity of the presented work.

\section{Preliminaries}

Here, some basic results are provided from the existing literature.

Definition 1 (see [24]). Let $s: R \rightarrow[0,1]$ satisfy the conditions, where $y_{0}, y_{1}, y_{2}, z \in R$ (set of real numbers)

(i) $s$ is upper semicontinuous

(ii) $s\left(\sigma y_{1}+(1-\sigma) y_{2}\right) \geq \min \left\{s\left(y_{1}\right), s\left(y_{2}\right)\right\}$

(iii) $\exists y_{0} \in R$ such that $\sigma\left(y_{0}\right)=1$

(iv) $\operatorname{cl}\{z \in R, \sigma(z)>0\}$ is compact

Then, $s$ is a fuzzy number.

Throughout this paper, $\widehat{F}_{R}$ represent the set that contains all fuzzy numbers.

Definition 2 (see [25]). The fuzzy number can be written as $(\underline{k}(q), \bar{k}(q))$ in order pair form, with $0 \leq q \leq 1$, and holds the following conditions:

(i) Nondecreasing bounded function, $\underline{s}(q)$ is leftcontinuous in $[0,1]$

(ii) Nonincreasing bounded function, $\bar{s}(q)$ is leftcontinuous in $[0,1]$

(iii) $\underline{s}(q) \leq \bar{s}(q)$

$q$ is a crisp nunmber when $\underline{s}(q)=\bar{s}(q)=q$.

Theorem 3. Let the mapping $F: Y \rightarrow Y$ is contractive with $k>1$, where $(Y, d)$ is a generalized complete metric space. If for $z \in Y, d\left(F^{n+1}(z), F^{n}(z)\right)<\infty$, with $n \geq 0$ and $F\left(z_{0}\right)=z_{0}$ $\in Y$, then

(i) $F^{n}(z)$ converge to $z_{0}$

(ii) The point $z_{0}$ is in $X_{0}=\left\{p \in Y \mid d\left(F_{n}(z), p\right)<\infty\right\}$

(iii) If $p \in Y$, then

$$
d\left(p, z_{0}\right) \leq \frac{1}{1-k} d(F(p), p)
$$

Definition 4. The mapping $d: Y_{1} \times Y_{1} \rightarrow R^{+}$is a generalized metric on $Y_{1}$ if and only if, $\forall x, y$ and $z \in Y_{1}$.

$\left(F_{1}\right) d(x, y)=0$ if and only if $x=y$

$\left(F_{2}\right) d(x, y)=d(x, y)$

$\left(F_{3}\right) d(x, z) \leq d(x, y)+d(y, z)$. Then, $\left(Y_{1}, d\right)$ is a metric space

Definition 5 (see [26]). The mapping $d_{H}: \widehat{F}_{R} \times \widehat{F}_{R} \rightarrow R^{+}$, defined by

$$
\begin{aligned}
d_{H}(x, y) & =\sup _{a_{1} \in[0,1]} d\left(x\left(a_{1}\right), y\left(a_{1}\right)\right) \\
& =\sup _{a_{1} \in[0,1]} \max \left\{\left|\underline{x}\left(a_{1}\right)-\underline{y}\left(a_{1}\right)\right|,\left|\bar{x}\left(a_{1}\right)-\bar{y}\left(a_{1}\right)\right|\right\} .
\end{aligned}
$$

The pair $\left(d_{H}, \widehat{F}_{R}\right)$ is a generalized complete metric space. Moreover, $\forall x_{1}, x_{2}, x_{3}, x_{4} \in \widehat{F}_{R}$ and $\alpha \in R+$ hold
$\left(D_{1}\right) d_{H}\left(x_{1}+x_{3}, x_{2}+x_{3}\right)=d_{H}\left(x_{1}, x_{2}\right)$
$\left(\mathrm{D}_{2}\right) d_{H}\left(\alpha x_{1}, \alpha x_{2}\right)=\alpha d_{H}\left(x_{1}, x_{2}\right)$
$\left(\mathrm{D}_{3}\right) d_{H}\left(x_{1}+x_{4}, x_{2}+x_{3}\right) \leq d_{H}\left(x_{1}, x_{2}\right)+d_{H}\left(x_{4}, x_{3}\right)$

Definition 6 (see [27]). The fuzzy function, $\chi:(a, b) \rightarrow \widehat{F}_{R}$, at $y \in(a, b)$, is generalized Hukuhara differentiable if there exist $\chi^{\prime}(y) \in \widehat{F}_{R}$ such that

(i) For $r>0$, sufficiently small, the Hukuhara difference, $\chi(y+r) \ominus \chi(y), \chi(y) \ominus \chi(y-r)$, and limits exist in the complete metric space $X \in \widehat{F}_{R}$

$$
\lim _{r \rightarrow 0} \frac{\chi(y) \ominus \chi(y+r)}{r}=\lim _{r \rightarrow 0} \frac{\chi(y-r) \ominus \chi(y)}{r}=\chi^{\prime}(y)
$$

(ii) For $r>0$, sufficiently small, the Hukuhara difference, $\chi(y) \ominus \chi(y+r), \chi(y-r) \ominus \chi(y)$, and limits exist in the complete metric space $X \in \widehat{F}_{R}$

$$
\lim _{r \rightarrow 0} \frac{\chi(y+r) \ominus \chi(y)}{(-r)}=\lim _{r \rightarrow 0} \frac{\chi(y) \ominus \chi(y-r)}{(-r)}=\chi^{\prime}(y)
$$

The first one is referred to (i)-differentiable and second one to (ii)-differentiable.

Definition 7 (see [27]). The fuzzy function, $\chi:(a, b) \rightarrow \widehat{F}_{R}$, at $y \in(a, b)$, is generalized Hukuhara second-order differentiable if there exist, $\chi^{\prime \prime}(y) \in \widehat{F}_{R}$ such that

(i) For $r>0$, sufficiently small, the Hukuhara difference, $\chi^{\prime}(y+r) \ominus \chi^{\prime}(y), \chi^{\prime}(y) \ominus \chi^{\prime}(y-r)$, and limits exist in the complete metric space $X \in \widehat{F}_{R}$ 


$$
\lim _{r \rightarrow 0} \frac{\chi^{\prime}(y) \ominus \chi^{\prime}(y+r)}{r}=\lim _{r \rightarrow 0} \frac{\chi^{\prime}(y-r) \ominus \chi^{\prime}(y)}{r}=\chi^{\prime \prime}(y)
$$

(ii) For $r>0$, sufficiently small, the Hukuhara difference, $\chi^{\prime}(y) \ominus \chi^{\prime}(y+r), \chi^{\prime}(y-r) \ominus \chi^{\prime}(y)$, and limits exist in the complete metric space $X \in \widehat{F}_{R}$

$$
\lim _{r \rightarrow 0} \frac{\chi^{\prime}(y+r) \ominus \chi^{\prime}(y)}{(-r)}=\lim _{r \rightarrow 0} \frac{\chi^{\prime}(y) \ominus \chi^{\prime}(y-r)}{(-r)}=\chi^{\prime \prime}(y)
$$

The first one is referred to (i)-differentiable and second one to (ii)-differentiable.

Definition 8 (see [28]). The crisp set $\{e \in R \mid \mu(e) \geq \gamma\}$ is the $\gamma$ -level set of fuzzy number $\mu \in \widehat{F}_{R}$. Moreover, the $\gamma$-level set is bounded and closed with upper and lower bond, $\bar{\mu}(q)$ and $\underline{\mu}(q)$, respectively, denoted by $(\underline{\mu}(q), \bar{\mu}(q))$.

Theorem 9 (see [29]). Let a continuous fuzzy function $\mu: R$ $\rightarrow F$, such that, $\mu(p)=(\mu(p), \bar{\mu}(p))$, and $0 \leq p \leq 1$ :

(i) $\mu(p)$ is (i)-differentiable, then $\mu^{\prime}(p)=\left(\mu^{\prime}(p), \bar{\mu}^{\prime}(p)\right)$

(ii) $\mu(p)$ is (ii)-differentiable, then $\mu^{\prime}(p)=\left(\bar{\mu}^{\prime}(p), \underline{\mu}^{\prime}(p)\right)$

Corollary 10 (see [26]). The continuous mapping $V: I \rightarrow \widehat{F}_{R}$ is integrable, where I is an interval.

Theorem 11 (see [27]). The generalized differentiable mapping $V: I \rightarrow \widehat{F}_{R}$ has integrable derivative $V^{\prime}$ on $I$.

(i) When $V$ is (i)-differentiable, $\forall x \in I$, we have

$$
V(x)=V\left(t_{0}\right)+\int_{t 0}^{x}(-1) V^{\prime}(t) d t
$$

(ii) When $V$ is (ii)-differentiable, $\forall x \in I$, we have

$$
V(x)=V\left(t_{0}\right)+\int_{t 0}^{x}(-1) V^{\prime}(t) d t
$$

Definition 12 (see [21]). The natural transform of $g(t)$ is $R(s, p)$ given by the following formula:

$$
R(s, p)=N\{g(t)\}=\int_{0}^{\infty} e^{-s t} g(p t) d t,
$$

where $s$ and $p$ are transform parameter which is real and positive.
Lemma 13 (see [22]). The duality relation of natural and Laplace transform is given by

$$
R(s, p)=N\{g(t)\}=\frac{1}{p} \int_{0}^{\infty} e^{-t / p} g(t) d t=\frac{1}{p} L\{g(t)\},
$$

where $L$ is the Laplace transform. The natural transform converts to Laplace transform by taking parameter $p=1$ and Sumudu transform by taking parameter $s=1$.

Definition 14 (see [23]). The natural transform of $n$th order derivative of $g(t)$ is

$$
N\left\{g^{n}(t)\right\}=\frac{s^{n}}{p^{n}} R(s, p)-\sum_{k=0}^{n-1} \frac{s^{n-(k-1)}}{p^{n-(k-1)}} \frac{1}{u} g^{k}(0) .
$$

The natural transform of $g^{\prime}(t)$ and $g^{\prime \prime}(t)$ first- and second-order derivative of $g(t)$ is given by

$$
\begin{aligned}
& N\left\{g^{\prime}(t)\right\}=\frac{s}{p} R(s, p)-\frac{1}{u} g(0), \\
& N\left\{g^{\prime \prime}(t)\right\}=\frac{s^{2}}{p^{2}} R(s, p)-\frac{s}{p^{2}} g(0)-\frac{1}{p} g^{\prime}(0) .
\end{aligned}
$$

\section{Existence, Uniqueness, and Stability Analysis}

Before dealing a physical model, it is important to know whether its solution exists. Now, if unique solution of a physical model exists, then the model is dealt properly. But if the solution is not unique then who is to say that the solution we found is what will actually happen in real life. Therefore, we need additional conditions to get unique solution. In this section, we carry out results for the existence of unique solutions of second-order fuzzy differential equations by using the contraction principle.

Let us consider FDEs

$$
\left\{\begin{array}{l}
u^{\prime \prime}(t)=F\left(t, u(t), u^{\prime}(t)\right), \\
u\left(t_{0}\right)=u_{0}=\left(\underline{u}_{0}, \bar{u}_{0}\right), \\
u^{\prime}\left(t_{0}\right)=u_{0}^{\prime}=\left(\underline{u}_{0}^{\prime}, \bar{u}_{0}^{\prime}\right),
\end{array}\right.
$$

where $Y=\left[t_{0}, t_{0}+T\right]$ and $F: Y \times \widehat{F_{R}} \times \widehat{F_{R}} \longrightarrow \widehat{F_{R}}$, and $u_{0}$, $u_{0}^{\prime} \in \widehat{F}_{R}$.

Definition 15. The fuzzy-valued function, $u \in C\left(Y, \widehat{F}_{R}\right)$, is the solution of differential Equation (13) if $u(t)$ satisfies Equation (13).

Let $u \in C(Y, \widehat{F} R)$, which is the solution of differential Equation (13), we reduce Equation (13) to an equivalent system of fuzzy-valued integral equations. Let $v: Y \rightarrow F^{\wedge} R$, which is continuous fuzzy-valued function, such that $u^{\prime}(t)$ $=v(t)$; therefore, we deduce 


$$
\left\{\begin{array}{l}
u^{\prime}(t)=v(t), \text { with } u\left(t_{0}\right)=u_{0}=\left(\underline{u}_{0}, \bar{u}_{0}\right), \\
v^{\prime}(t)=F(t, u(t), v(t)), v\left(t_{0}\right)=u_{0}^{\prime}=\left(\underline{u}_{0}^{\prime}, \bar{u}_{0}^{\prime}\right) .
\end{array}\right.
$$

Using Corollary 10, Theorem 11, and initial condition, then the following four systems of equations are obtained:

(1) If $u(t)$ and $u^{\prime}(t)=v(t)$ are (i)-differentiable.

$$
\left\{\begin{array}{l}
u(t)=u_{0}+\int_{t_{0}}^{t} v(s) d s, \\
v(t)=u_{0}^{\prime}+\int_{t_{0}}^{t} F(s, u(s), v(s)) d s
\end{array}\right.
$$

(2) If $u(t)$ is (i)-differentiable and $u^{\prime}(t)=v(t)$ is (ii)differentiable.

$$
\left\{\begin{array}{l}
u(t)=u_{0}+\int_{t_{0}}^{t} v(s) d s \\
v(t)=u_{0}^{\prime} \ominus \int_{t_{0}}^{t}(-1) F(s, u(s), v(s)) d s
\end{array}\right.
$$

(3) If $u(t)$ is (ii)-differentiable and $u^{\prime}(t)=v(t)$ is (i)differentiable.

$$
\left\{\begin{array}{l}
u(t)=u_{0} \ominus \int_{t_{0}}^{t}(-1) v(s) d s, \\
v(t)=u_{0}^{\prime}+\int_{t_{0}}^{t} F(s, u(s), v(s)) d s
\end{array}\right.
$$

(4) If $u(t)$ and $u^{\prime}(t)=v(t)$ are (ii)-differentiable.

$$
\left\{\begin{array}{l}
u(t)=u_{0} \ominus \int_{t_{0}}^{t}(-1) v(s) d s, \\
v(t)=u_{0}^{\prime} \ominus \int_{t_{0}}^{t}(-1) F(s, u(s), v(s)) d s
\end{array}\right.
$$

Theorem 16. Let the continuous fuzzy-valued function $u \in$ $C\left(Y, \widehat{F}_{R}\right)$ and $\chi=C\left(Y, F \wedge_{R}\right)^{2}$ such that $H: \chi \times \chi \rightarrow R^{+} \cup$ $\{+\infty\}$, with

$$
\begin{aligned}
H\left(\left(u_{1}, v_{1}\right),\left(u_{2}, v_{2}\right)\right)= & \inf \left\{N \in R^{+} \cup\{+\infty\}:\left(d_{H}\left(u_{1}, u_{2}\right)\right.\right. \\
& \left.\left.+d_{H}\left(v_{1}, v_{2}\right)\right) e^{-C\left(t-t_{0}\right)} \leq N \widehat{\phi}(t), \forall t \in Y\right\}
\end{aligned}
$$

where $\widehat{\phi}: \widehat{Y} \rightarrow(0, \infty)$ be continuous mapping; then, $(\chi, H)$ is a generalized complete metric space.

Proof. The first two conditions $F_{1}$ and $F_{2}$ are easy to show; therefore, we only show $F_{3}$. Assume that for every $\left(u_{1}, v_{1}\right)$, $\left(u_{2}, v_{2}\right),\left(u_{3}, v_{3}\right) \in \chi$, there exist $t \in Y$, such that

$$
\begin{aligned}
H\left(\left(u_{1}, v_{1}\right),\left(u_{3}, v_{3}\right)\right)= & \left(d_{H}\left(u_{1}, u_{3}\right)+d_{H}\left(v_{1}, v_{3}\right)\right) e^{-C\left(t-t_{0}\right)} \\
\leq & \left(d_{H}\left(u_{1}, u_{2}\right)+d_{H}\left(u_{2}, u_{3}\right)\right) e^{-C\left(t-t_{0}\right)} \\
& +\left(d_{H}\left(v_{1}, v_{2}\right)+d_{H}\left(v_{2}, v_{3}\right)\right) e^{-C\left(t-t_{0}\right)} \\
= & \left(d_{H}\left(u_{1}, u_{2}\right)+d_{H}\left(v_{1}, v_{2}\right)\right) e^{-C\left(t-t_{0}\right)} \\
& +\left(d_{H}\left(u_{2}, u_{3}\right)+d_{H}\left(v_{2}, v_{3}\right)\right) e^{-C\left(t-t_{0}\right)}, \\
H\left(\left(u_{1}, v_{1}\right),\left(u_{3}, v_{3}\right)\right) \leq & H\left(\left(u_{1}, v_{1}\right),\left(u_{2}, v_{2}\right)\right)+H\left(\left(u_{2}, v_{2}\right),\left(u_{3}, v_{3}\right)\right) .
\end{aligned}
$$

Hence, $(\chi, H)$ is a generalized metric space.

Now, we need to show that $(\chi, H)$ is complete. Let us consider the Cauchy sequence, $\left(u_{n}, v_{n}\right)$ on $(\chi, H)$, then $H\left(\left(u_{n}, v_{n}\right),\left(u_{m}, v_{m}\right) \leq \varepsilon\right.$, for all $n, m \geq N(\varepsilon)$, using definition, (19), one can get

$$
\left(d_{H}\left(u_{n}, u_{m}\right)+d_{H}\left(v_{n}, v_{m}\right)\right) e^{-C\left(t-t_{0}\right)} \leq \epsilon \widehat{\phi}(t), \quad \forall n, m \geq N(\varepsilon), t \in Y
$$

Since $\left(\widehat{F}_{R}, d_{H}\right)$ is a complete metric space, then there is $\exists u(t), v(t): Y \rightarrow \widehat{F}_{R}$, such that Cauchy sequence, $u_{n}(t)$ and $v_{n}(t)$ converges to $u(t)$ and $v(t)$, respectively.

When $m \rightarrow \infty$, then (21) produced

$$
\begin{aligned}
&\left(d_{H}\left(u_{n}, u(t)\right)+d_{H}\left(v_{n}, v(t)\right)\right) e^{-C\left(t-t_{0}\right)} \leq \epsilon \widehat{\phi}(t), \forall n, \geq N(\varepsilon), t \in Y, \\
& H\left(\left(u_{n}(t), v_{n}(t)\right),(u(t), v(t))\right) \leq \varepsilon \widehat{\phi}(t), \quad \forall n, \geq N(\varepsilon), t \in Y .
\end{aligned}
$$

Hence, $\left(u_{n}(t), v_{n}(t)\right)$ converge to $(u(t), v(t))$, in $(\chi, H)$. Therefore, $(\chi, H)$ is a complete generalized metric space.

Theorem 17. The fuzzy problem (13) has a unique and HyersUlam stable solution $\widehat{u}, \widehat{v}: Y \rightarrow \widehat{F}_{R}$, defined by

$$
\left\{\begin{array}{l}
\widehat{u}=u_{0}+\int_{t_{0}}^{t} v(s) d s, \\
\widehat{v}=u_{0}^{\prime}+\int_{t_{0}}^{t} F(s, u(s), v(s)) d s .
\end{array}\right.
$$

If the following conditions hold for some nonnegative $L, \varepsilon$ and $T=t-t_{0}$. 
(i) $d_{H}\left(F\left(t, u_{1}, v_{1}\right), F\left(t, u_{2}, v_{2}\right) \leq L d_{H}\left(u_{1}, u_{2}\right)+(L-1) d_{H}\right.$ $\left(v_{1}, v_{2}\right)$,

where $F: Y \times \widehat{F}_{R} \times \widehat{F}_{R} \rightarrow \widehat{F}_{R}$ be fuzzy-valued continuous function $\forall u_{1}, v_{1}, u_{2}, v_{2} \in \widehat{F}_{R}$ and $t \in Y$

(ii) $d_{H}(D u(t), v(t))+d_{H}(D v(t), F(t, u(t), v(t))) \leq \varepsilon$,

where $u, v: Y \rightarrow \widehat{F}_{R}$ are (i)-differentiable

(iii) $d_{H}(u, \widehat{u}) \leq(1+L) \varepsilon T$ and $d_{H}(v, \widehat{v}) \leq(1+L) \varepsilon T$

Proof. Let $C\left(Y, \widehat{F}_{R}\right)=\left\{u: Y \rightarrow \widehat{F}_{R}\right.$, be continuous fuzzy valued function $\}$ and $\chi=C\left(Y, F \wedge_{R}\right)^{2}$ such that $H: \chi \times \chi$ $\rightarrow R^{+} \cup\{+\infty\}$, with

$$
\begin{aligned}
H\left(\left(u_{1}, v_{1}\right),\left(u_{2}, v_{2}\right)\right)= & \inf \left\{N \in R^{+} \cup\{+\infty\}:\left(d_{H}\left(u_{1}, u_{2}\right)\right.\right. \\
& \left.\left.+d_{H}\left(v_{1}, v_{2}\right)\right) e^{-(L+1)\left(t-\mathrm{t}_{0}\right)} \leq N \widehat{\phi}(t) \forall t \in Y\right\},
\end{aligned}
$$

where $\widehat{\phi}: \widehat{Y} \rightarrow(0, \infty)$ be continuous mapping, then $(\chi, H)$ is a generalized complete metric space, in view of Theorem 16. Let us define a self operator $G: \chi \rightarrow \chi, \forall(u, v)$ $\in \chi$ and $t \in Y$, by

$$
\begin{aligned}
G(u(t), v(t)) & =(G(u), G(v)) \\
& =\left(u_{0}+\int_{t_{0}}^{t} v(s) d s, u_{0}^{\prime}+\int_{t_{0}}^{t} F(s, u(s), v(s) d s) d s\right) .
\end{aligned}
$$

First, we show $G(u, v) \in \chi$. Let $\left(p_{0}, q_{0}\right) \in \chi$ and $t \in Y$,

$$
\begin{array}{r}
\left\{d_{H}\left(G\left(p_{0}\right), p_{0}\right)+d_{H}\left(G\left(q_{0}\right), q_{0}\right)\right\} e^{-(L+1)\left(t-t_{0}\right)}<\infty, \\
H\left(\left(G\left(p_{0}, q_{0}\right),\left(p_{0}, q_{0}\right)\right)<\infty .\right.
\end{array}
$$

Hence, $G(u(t), v(t))=(G(u), G(v))$ is well defined. Now, we can show for all $(u, v) \in \chi$, the operator $G$ is strictly contractive on $(\chi, H)$.

$$
\begin{aligned}
d_{H}( & \left.G\left(u_{1}\right), G\left(u_{2}\right)\right)+d_{H}\left(G\left(v_{1}\right), G\left(v_{2}\right)\right) \\
= & d_{H}\left(u_{0}+\int_{t_{0}}^{t} v_{1}(s) d s, u_{0}+\int_{t_{0}}^{t} v_{2}(s) d s\right) \\
& +d_{H}\left(u_{0}^{\prime}+\int_{t_{0}}^{t} F\left(s, u_{1}(s), v_{1}\right)(s) d s, u_{0}^{\prime}+\int_{t_{0}}^{t} F\left(s, u_{2}(s), v_{2}\right)(s) d s\right) \\
= & d_{H}\left(\int_{t_{0}}^{t} v_{1}(s) d s, \int_{t_{0}}^{t} v_{2}(s) d s\right) \\
& +d_{H}\left(\int_{t_{0}}^{t} F\left(s, u_{1}(s), v_{1}(s)\right) d s, \int_{t_{0}}^{t} F\left(s, u_{2}(s), v_{2}(s)\right) d s\right) \\
\leq & L \int_{t_{0}}^{t}\left(d_{H}\left(u_{1}, u_{2}\right)+d_{H}\left(v_{1}, v_{2}\right)\right) e^{-(L+1)\left(s-t_{0}\right)} e^{(L+1)\left(s-t_{0}\right)} d s \\
= & \frac{L}{L+1} H\left(\left(u_{1}, v_{1}\right),\left(u_{2}, v_{2}\right)\right) e^{(L+1)\left(t-t_{0}\right)} .
\end{aligned}
$$

To multiply $e^{-(L+1)\left(t-t_{0}\right)}$, on both sides of the above inequality, we have

$$
\begin{aligned}
& \left(d_{H}\left(G\left(u_{1}\right), G\left(u_{2}\right)\right)+d_{H}\left(G\left(v_{1}\right), G\left(v_{2}\right)\right)\right) e^{-(L+1)\left(t-t_{0}\right)} \\
& \quad \leq \frac{L}{L+1} H\left(\left(u_{1}, v_{1}\right),\left(u_{2}, v_{2}\right)\right) .
\end{aligned}
$$

Now, from the definition of $H$, for all $t \in Y$, one can get

$$
H\left(G\left(u_{1}, v_{1}\right), G\left(u_{2}, v_{2}\right)\right) \leq \frac{L}{L+1} H\left(\left(u_{1}, v_{1}\right),\left(u_{2}, v_{2}\right)\right)
$$

This indicates that $G$ is strictly contractive on $(\chi, H)$. Therefore, there exist unique solution of problem (13).

The $u(t) \ominus u\left(t_{0}\right)$ and $v(t) \ominus u^{\prime}\left(t_{0}\right)$, Hukuhara differences exist for $t \in Y$, where $u$ and $v$ are (i)-differentiable, then using condition (ii), we have

$$
\begin{aligned}
d_{H}(u, G(u))+d_{H}(v, G(v))= & d_{H}\left(u(t), u_{0}+\int_{t_{0}}^{t} v(s) d s\right)+d_{H} \\
& \cdot\left(v(t), u_{0}^{\prime}+\int_{t_{0}}^{t} F(s, u(s), v(s)) d s\right) \\
= & d_{H}\left(u(t)-u_{0}, \int_{t_{0}}^{t} v(s) d s\right)+d_{H} \\
& \cdot\left(v(t)-u_{0}^{\prime}, \int_{t_{0}}^{t} F(s, u(s), v(s)) d s\right) \\
= & d_{H}\left(\int_{t_{0}}^{t} D u(t), \int_{t_{0}}^{t} v(s) d s\right)+d_{H} \\
& \cdot\left(\int_{t_{0}}^{t} D v(t), \int_{t_{0}}^{t} F(s, u(s), v(s)) d s\right) \\
= & \int_{t_{0}}^{t}\left\{d_{H}(D u(t), v(t))+d_{H}\right. \\
& \left.\cdot\left(D^{2} u(t), F(t, u(t), v(t))\right)\right\} d t \\
\leq & \varepsilon\left(t-t_{0}\right) .
\end{aligned}
$$

To multiply $e^{-(L+1)\left(t-t_{0}\right)}$, on both sides of the above inequality, we have

$$
\left(d_{H}(u, G(u))+d_{H}(v, G(v)) e^{-(L+1)\left(t-t_{0}\right)} \leq \varepsilon\left(t-t_{0}\right) e^{-(L+1)\left(t-t_{0}\right)}\right.
$$

Now, from the definition of $H$ and substitute $T=t-t_{0}$, for all $t \in Y$, we can obtain

$$
H((u, v), G(u, v)) \leq \varepsilon T e^{-(L+1)\left(t-t_{0}\right)} .
$$

From, Theorem 3 condition (iii), there exist unique solution $(\widehat{u}, \widehat{v})$, of problem (13), such that 
$H((u, v),(\widehat{u}, \widehat{v}))=\frac{1}{1-} H((u, v), G(u, v)) \leq(1+L) \varepsilon T e^{-(L+1)\left(t-t_{0}\right)}$.

Using definition of $H$, one can get

$$
\left.d_{H}(u, \widehat{u})+d_{H}(v, \widehat{v})\right) \leq(1+L) \varepsilon T, \quad \forall t \in Y .
$$

Hence, for all $t \in Y$, we can get

$$
\begin{aligned}
& d_{H}(u, \widehat{u}) \leq(1+L) \varepsilon T \\
& d_{H}(v, \widehat{v}) \leq(1+L) \varepsilon T .
\end{aligned}
$$

Hence, the fuzzy solutions of the problem (13) are HyersUlam stable.

Theorem 18. Let $F: Y \times \widehat{F}_{R} \times \widehat{F}_{R} \rightarrow \widehat{F}_{R}$ be the fuzzy-valued continuous function such that

$d_{H}\left(F\left(t, u_{1}, v_{1}\right), F\left(t, u_{2}, v_{2}\right)\right) \leq d_{H}\left(u_{1}, u_{2}\right), \quad \forall u_{1}, v_{1}, u_{2}, v_{2} \in \widehat{F}_{R}, t \in Y$.

If the functions, $u: Y \rightarrow \widehat{F}_{R}$, are (i)-differentiable and $v: Y \rightarrow \widehat{F}_{R}$ is (ii)-differentiable, the following condition holds:

$d_{H}(D u(t), v(t))+d_{H}(D v(t), F(t, u(t), v(t))) \leq \varepsilon$, some nonnegative, $\varepsilon$.

Then, there exist unique solution of the problem (13), $\widehat{u}$, $\widehat{v}: Y \rightarrow \widehat{F}_{R}$, defined by

$$
\left\{\begin{array}{l}
\widehat{u}=u_{0}+\int_{t_{0}}^{t} v(s) d s \\
\widehat{v}=u_{0}^{\prime}(-1) \Theta \int_{t_{0}}^{t} F(s, u(s), v(s)) d s
\end{array}\right.
$$

The fuzzy solution is Hyers-Ulam stable, if the following conditions are satisfied:

$$
\begin{aligned}
& d_{H}(u, \widehat{u}) \leq(L+1) \varepsilon T, \\
& d_{H}(v, \widehat{v}) \leq(L+1) \varepsilon T,
\end{aligned}
$$

where $L$ is nonnegative constant and $T=t-t_{0}$.

Proof. The proof can be easily obtained on the similar procedure of Theorem 17.

Theorem 19. Let $F: Y \times \widehat{F}_{R} \times \widehat{F}_{R} \rightarrow \widehat{F}_{R}$, be the fuzzy-valued continuous function such that

$d_{H}\left(F\left(t, u_{1}, v_{1}\right), F\left(t, u_{2}, v_{2}\right) \leq d_{H}\left(u_{1}, u_{2}\right), \quad \forall u_{1}, v_{1}, u_{2}, v_{2} \in \widehat{F}_{R}, t \in Y\right.$.
If the function $u: Y \rightarrow \widehat{F}_{R}$ is (ii)-differentiable and $v: Y$ $\rightarrow \widehat{F}_{R}$ is (i)-differentiable, the following condition holds:

$d_{H}(D u(t), v(t))+d_{H}(D v(t), F(t, u(t), v(t))) \leq \varepsilon$, some nonnegative, $\varepsilon$.

Then, there exist unique solutions of the problem (13), $\widehat{u}$, $\widehat{v}: Y \rightarrow \widehat{F}_{R}$, defined by

$$
\left\{\begin{array}{l}
\widehat{u}=u_{0}(-1) \Theta \int_{t_{0}}^{t} v(s) d s \\
\widehat{v}=u_{0}^{\prime}+\int_{t_{0}}^{t} F(s, u(s), v(s)) d s .
\end{array}\right.
$$

The fuzzy solution is Hyers-Ulam stable, if the following conditions are satisfied:

$$
\begin{aligned}
& d_{H}(u, \widehat{u}) \leq(L+1) \varepsilon T, \\
& d_{H}(v, \widehat{v}) \leq(L+1) \varepsilon T,
\end{aligned}
$$

where $L$ is nonnegative constant and $T=t-t_{0}$.

Proof. The proof can be easily obtained on the similar procedure of Theorem 17.

Theorem 20. Let $F: Y \times \widehat{F}_{R} \times \widehat{F}_{R} \rightarrow \widehat{F}_{R}$ be the fuzzy-valued continuous function such that

$d_{H}\left(F\left(t, u_{1}, v_{1}\right), F\left(t, u_{2}, v_{2}\right) \leq d_{H}\left(u_{1}, u_{2}\right), \quad \forall, u_{1}, v_{1}, u_{2}, v_{2} \in \widehat{F}_{R}, t \in Y\right.$.

If the functions $u, v: Y \rightarrow \widehat{F}_{R}$ are (ii)-differentiable, the following conditions hold:

$d_{H}(D u(t), v(t))+d_{H}(D v(t), F(t, u(t), v(t))) \leq \varepsilon$, some nonnegative, $\varepsilon$.

Then, there exist unique solution of the problem (13), $\widehat{u}$, $\widehat{v}: Y \rightarrow \widehat{F}_{R}$, defined by

$$
\left\{\begin{array}{l}
\widehat{u}=u_{0}+\int_{t_{0}}^{t} v(s) d s, \\
\widehat{v}=u_{0}^{\prime}+\int_{t_{0}}^{t} F(s, u(s), v(s)) d s .
\end{array}\right.
$$

The fuzzy solution is Hyers-Ulam stable, if the following conditions are satisfied:

$$
\begin{aligned}
& d_{H}(u, \widehat{u}) \leq(L+1) \varepsilon T, \\
& d_{H}(v, \widehat{v}) \leq(L+1) \varepsilon T,
\end{aligned}
$$

where $L$ is nonnegative constant and $T=t-t_{0}$. 
Proof. Using a procedure similar to Theorem 17, one can easily prove.

\section{Natural Transforms and Solutions of the Proposed Fuzzy Differential Equations}

Theorem 21. Let fuzzy-valued function $\chi(w)$ be continuous such that $e^{-s w} \chi(u w), e^{-s w} \chi^{\prime}(u w)$, and $e^{-s w} \chi^{\prime \prime}(u w)$ exist and are continuous. Moreover, they are Riemann integrable on $[0, \infty)$; then,

(A) If $\chi(w)$ and $\chi^{\prime}(w)$ are (i)-differentiable, then

$$
\mathscr{N}\left\{\chi^{\prime \prime}(w)\right\}=\left[\frac{s^{2}}{u^{2}} \widehat{R}(s, u) \ominus \frac{s}{u^{2}} \chi(0)\right] \ominus \frac{1}{u} \chi^{\prime}(0)
$$

(B) If $\chi(w)$ is (i)-differentiable and $\chi^{\prime}(w)$ is (ii)-differentiable, then

$\mathcal{N}\left\{\chi^{\prime \prime}(w)\right\}=\left(-\frac{1}{u} \chi^{\prime}(0)\right) \ominus\left[\left(-\frac{s^{2}}{u^{2}} \widehat{R}(s, u)\right) \ominus\left(-\frac{s}{u^{2}} \chi(0)\right)\right]$

(C) If $\chi(w)$ is (ii)-differentiable and $\chi^{\prime}(w)$ is (i)-differentiable, then

$\mathcal{N}\left\{\chi^{\prime \prime}(w)\right\}=\left[\left(-\frac{s}{u^{2}} \chi(0)\right) \ominus\left(-\frac{s^{2}}{u^{2}} \widehat{R}(s, u)\right)\right] \ominus \frac{1}{u} \chi^{\prime}(0)$.

(D) If $\chi(w)$ and $\chi^{\prime}(w)$ are (ii)-differentiable, then

$$
\mathcal{N}\left\{\chi^{\prime \prime}(w)\right\}=\left(-\frac{1}{u} \chi^{\prime}(0)\right) \ominus\left[\frac{s}{u^{2}} \chi(0) \ominus \frac{s^{2}}{u^{2}} \widehat{R}(s, u)\right]
$$

Proof.

(A) Let $\chi(w)$ and $\chi^{\prime}(w)$ are (i)-differentiable, then

$$
\mathcal{N}\left\{\chi^{\prime}(w)\right\}=\frac{s}{u}[\widehat{R}(s, u)] \ominus \frac{1}{u} \chi(0),
$$

and $\mathcal{N}\left\{\chi^{\prime \prime}(w)\right\}=\frac{s}{u}\left[\mathcal{N}\left\{\chi^{\prime}(w)\right\}\right] \ominus \frac{1}{u} \chi^{\prime}(0)$.
Using (52), this identity yields

$$
\begin{aligned}
& \mathcal{N}\left\{\chi^{\prime \prime}(w)\right\}=\frac{s}{u}\left[\frac{s}{u}[\widehat{R}(s, u)] \ominus \frac{1}{u} \chi(0)\right] \ominus \frac{1}{u} \chi^{\prime}(0), \\
& \mathcal{N}\left\{\chi^{\prime \prime}(w)\right\}=\left[\frac{s^{2}}{u^{2}} \widehat{R}(s, u) \ominus \frac{s}{u^{2}} \chi(0)\right] \ominus \frac{1}{u} \chi^{\prime}(0)
\end{aligned}
$$

(B) If, $\chi(w)$ and $\chi^{\prime}(w)$ are (i)-differentiable and (ii)-differentiable, respectively, then

$$
\begin{aligned}
& \mathcal{N}\left\{\chi^{\prime}(w)\right\}=\frac{s}{u}[\widehat{R}(s, u)] \ominus \frac{1}{u} \chi(0) \\
& \mathcal{N}\left\{\chi^{\prime \prime}(w)\right\}=\left(-\frac{1}{u} \chi^{\prime}(0)\right) \ominus\left(-\frac{s}{u}\left[\mathcal{N}\left\{\chi^{\prime}(w)\right\}\right]\right) .
\end{aligned}
$$

Using (54), this identity yields

$$
\begin{aligned}
& \mathcal{N}\left\{\chi^{\prime \prime}(w)\right\}=\left(-\frac{1}{u} \chi^{\prime}(0)\right) \ominus\left[-\frac{s}{u}\left(\frac{s}{u}[\widehat{R}(s, u)] \ominus \frac{1}{u} \chi(0)\right)\right], \\
& \mathcal{N}\left\{\chi^{\prime \prime}(w)\right\}=\left(-\frac{1}{u} \chi^{\prime}(0)\right) \ominus\left[\left(-\frac{s^{2}}{u^{2}} \widehat{R}(s, u)\right) \ominus\left(-\frac{s}{u^{2}} \chi(0)\right)\right]
\end{aligned}
$$

(C) If $\chi^{\prime}(w)$ and $\chi(w)$ are (i)-differentiable and (ii)-differentiable, respectively, then

$$
\begin{aligned}
& \mathcal{N}\left\{\chi^{\prime}(w)\right\}=\left(-\frac{1}{u} \chi(0)\right) \ominus\left(-\frac{s}{u}[\widehat{R}(s, u)]\right), \\
& \mathcal{N}\left\{\chi^{\prime \prime}(w)\right\}=\frac{s}{u}\left[\mathcal{N}\left\{\chi^{\prime}(w)\right\}\right] \ominus \frac{1}{u} \chi(0) .
\end{aligned}
$$

Using (56), this identity yields

$$
\begin{aligned}
& \left.\mathcal{N}\left\{\chi^{\prime \prime}(w)\right\}=\left(-\frac{s}{u^{2}} \chi^{\prime}(0)\right) \ominus\left(-\frac{s^{2}}{u^{2}}[\widehat{R}(s, u)]\right) \ominus \frac{1}{u} \chi(0)\right), \\
& \mathcal{N}\left\{\chi^{\prime \prime}(w)\right\}=\left[\left(-\frac{1}{u} \chi(0)\right) \ominus\left(-\frac{s^{2}}{u^{2}} \widehat{R}(s, u)\right)\right] \ominus \frac{1}{u} \chi^{\prime}(0)
\end{aligned}
$$




$$
\begin{aligned}
& \mathcal{N}\left\{\chi^{\prime}(w)\right\}=\left(-\frac{1}{u} \chi(0)\right) !\left(-\frac{s}{u}[\widehat{R}(s, u)]\right) \\
& \mathcal{N}\left\{\chi^{\prime \prime}(w)\right\}=\left(-\frac{1}{u} \chi^{\prime}(0)\right) \ominus\left(-\frac{s}{u}\left[\mathcal{N}\left\{\chi^{\prime}(w)\right\}\right]\right) .
\end{aligned}
$$

Using (58), this identity yields

$$
\begin{aligned}
& \mathcal{N}\left\{\chi^{\prime \prime}(w)\right\}=\left(-\frac{1}{u} \chi^{\prime}(0)\right) \ominus\left[-\frac{s}{u}\left(\left(-\frac{1}{u} \chi^{\prime}(0)\right) \ominus\left(-\frac{s}{u}[\widehat{R}(s, u)]\right)\right)\right], \\
& \left.\mathcal{N}\left\{\chi^{\prime \prime}(w)\right\}=\left(-\frac{1}{u} \chi^{\prime}(0)\right) \ominus\left[\frac{s}{u^{2}} \chi(0) \ominus \frac{s^{2}}{u^{2}} \widehat{R}(s, u)\right)\right]
\end{aligned}
$$

Next, we have to solve problem (13), with natural transform. The fuzzy solution of the concerned problem can be discussed in four cases. Taking natural transform of (13), we can get

$$
\mathcal{N}\left[F\left(w, \chi(w), \chi^{\prime}(w)\right)\right]=\mathcal{N}\left\{\chi^{\prime \prime}(w)\right\}
$$

Case I. Let $\chi(w)$ and $\chi^{\prime}(w)$ are (i)-differentiable; then, using Theorem 21, in (60), one can get

$$
\left\{\begin{array}{l}
\mathcal{N}\left[\underline{F}\left(w, \chi(w), \chi^{\prime}(w), \gamma\right)\right]=\left[\frac{s^{2}}{u^{2}} \underline{\hat{R}}(s, u) \ominus \frac{s}{u^{2}} \underline{\chi}(0, \gamma)\right] \ominus \frac{1}{u} \underline{\chi}^{\prime}(0, \gamma), \\
\mathcal{N}\left[\bar{F}\left(w, \chi(w), \chi^{\prime}(w), \gamma\right)\right]=\left[\frac{s^{2}}{u^{2}} \widehat{R}(s, u) \ominus \frac{s}{u^{2}} \bar{\chi}(0, \gamma)\right] \ominus \frac{1}{u} \bar{\chi}^{\prime}(0, \gamma),
\end{array}\right.
$$

where

$$
\begin{aligned}
\underline{F}\left(w, \chi(w), \chi^{\prime}(w), \gamma\right)= & \min \left\{F\left(w, \chi(w), \chi^{\prime}(w)\right) \mid \chi(w) \epsilon\right. \\
& \cdot(\underline{\chi}(w, \gamma), \bar{\chi}(w, \gamma)) ; \chi^{\prime}(w) \epsilon \\
& \left.\cdot\left(\underline{\chi^{\prime}}(w, \gamma), \bar{\chi}^{\prime}(w, \gamma)\right)\right\}, \\
\bar{F}\left(w, \chi(w), \chi^{\prime}(w), \gamma\right)= & \max \left\{F\left(w, \chi(w), \chi^{\prime}(w)\right) \mid \chi(w) \epsilon\right. \\
& \cdot(\underline{\chi}(w, \gamma), \bar{\chi}(w, \gamma)) ; \chi^{\prime}(w) \epsilon \\
& \left.\cdot\left(\underline{\chi}^{\prime}(w, \gamma), \bar{\chi}^{\prime}(w, \gamma)\right)\right\} .
\end{aligned}
$$

Assume that solving Equation (61), satisfying the conditions $\chi\left(w_{0}\right)=\chi_{0}=\left(\underline{\chi}_{0}, \bar{\chi}_{0}\right)$ and $\chi^{\prime}\left(w_{0}\right)=\chi_{0}^{\prime}=\left(\underline{\chi}_{0}^{\prime}, \bar{\chi}_{0}^{\prime}\right)$, one can obtain the solutions as follows:

$$
\left\{\begin{array}{l}
\underline{\widehat{R}}(s, u, \gamma)=R_{1}(s, u, \gamma), \\
\widehat{\bar{R}}(s, u, \gamma)=G_{1}(s, u, \gamma) .
\end{array}\right.
$$

Taking inverse fuzzy natural transform of Equation (63), one can find $\chi(w, \gamma)$ and $\bar{\chi}(w, \gamma)$, as follows:

$$
\left\{\begin{array}{l}
\underline{\chi}(w, \gamma)=\mathcal{N}^{-1}\left\{R_{1}(s, u, \gamma)\right\} \\
\bar{\chi}(w, \gamma)=\mathcal{N}^{-1}\left\{G_{1}(s, u, \gamma)\right\}
\end{array}\right.
$$

Case II. Let $\chi(w)$ is (i)-differentiable and $\chi^{\prime}(w)$ is (ii)-differentiable, then using Theorem 21, in (60), one can get

$\left\{\begin{array}{l}\mathcal{N}\left[\underline{F}\left(w, \chi(w), \chi^{\prime}(w), \gamma\right)\right]=\left(-\frac{1}{u} \bar{\chi}^{\prime}(0, \gamma)\right) \ominus\left[\left(-\frac{s^{2}}{u^{2}} \underline{\widehat{R}}(s, u, \gamma)\right) \ominus\left(-\frac{s}{u^{2}} \underline{\chi}(0, \gamma)\right)\right], \\ \mathcal{N}\left[\bar{F}\left(w, \chi(w), \chi^{\prime}(w), \gamma\right)\right]=\left(-\frac{1}{u} \underline{\chi}^{\prime}(0, \gamma)\right) \ominus\left[\left(-\frac{s^{2}}{u^{2}} \widehat{\bar{R}}(s, u, \gamma)\right) \ominus\left(-\frac{s}{u^{2}} \bar{\chi}(0, \gamma)\right)\right] .\end{array}\right.$

Using initial condition in (65), $\chi\left(w_{0}\right)=\chi_{0}=\left(\underline{\chi}_{0}, \bar{\chi}_{0}\right)$ and $\chi^{\prime}\left(w_{0}\right)=\chi_{0}^{\prime}=\left(\bar{\chi}_{0}^{\prime}, \underline{\chi}_{0}^{\prime}\right)$, one can get

$\left\{\begin{array}{l}\mathcal{N}\left[\underline{F}\left(w, \chi(w), \chi^{\prime}(w), \gamma\right)\right]=\left[\frac{s^{2}}{u^{2}} \underline{\underline{R}}(s, u, \gamma) \ominus \frac{s}{u^{2}} \underline{\chi}_{0}(\gamma)\right] \ominus \frac{1}{u} \bar{\chi}_{0}^{\prime}(\gamma), \\ \mathcal{N}\left[\bar{F}\left(w, \chi(w), \chi^{\prime}(w), \gamma\right)\right]=\left[\frac{s^{2}}{u^{2}} \widehat{\bar{R}}(s, u, \gamma) \ominus \frac{s}{u^{2}} \bar{\chi}_{0}(\gamma)\right] \ominus \frac{1}{u} \underline{\chi}_{0}^{\prime}(\gamma) .\end{array}\right.$

Assume that by solving Equation (66), we can get the solutions as follows:

$$
\left\{\begin{array}{l}
\underline{\widehat{R}}(s, u, \gamma)=R_{2}(s, u, \gamma), \\
\widehat{\bar{R}}(s, u, \gamma)=G_{2}(s, u, \gamma) .
\end{array}\right.
$$

Taking inverse fuzzy natural transform of Equation (67), one can find $\underline{\chi}(w, \gamma)$ and $\bar{\chi}(w, \gamma)$,

$$
\left\{\begin{array}{l}
\underline{\chi}(w, \gamma)=\mathcal{N}^{-1}\left\{R_{2}(s, u, \gamma)\right\} \\
\bar{\chi}(w, \gamma)=\mathcal{N}^{-1}\left\{G_{2}(s, u, \gamma)\right\} .
\end{array}\right.
$$

Case III. Let $\chi(w)$ is (ii)-differentiable and $\chi^{\prime}(w)$ is (i)-differentiable, then using Theorem 21 , in (60), one can get

$$
\left\{\begin{array}{l}
\mathcal{N}\left[\bar{F}\left(w, \chi(w), \chi^{\prime}(w), \gamma\right)\right]=\left[\left(-\frac{s}{u^{2}} \bar{\chi}(0, \gamma)\right) \ominus\left(-\frac{s^{2}}{u^{2}} \widehat{\hat{R}}(s, \mathrm{u}, \gamma)\right)\right] \ominus\left(-\frac{1}{u} \underline{\chi}^{\prime}(0, \gamma)\right), \\
\mathcal{N}\left[\underline{F}\left(w, \chi(w), \chi^{\prime}(w), \gamma\right)\right]=\left[\left(-\frac{s}{u^{2}} \underline{\chi}(0, \gamma)\right) \ominus\left(-\frac{s^{2}}{u^{2}} \underline{\widehat{R}}(s, u, \gamma)\right)\right] \ominus\left(-\frac{1}{u} \bar{\chi}^{\prime}(0, \gamma)\right) .
\end{array}\right.
$$

Using initial condition in (69), $\chi\left(w_{0}\right)=\chi_{0}=\left(\bar{\chi}_{0}, \underline{\chi}_{0}\right)$ and $\chi^{\prime}\left(w_{0}\right)=\chi_{0}^{\prime}=\left(\underline{\chi}_{0}^{\prime}, \bar{\chi}_{0}^{\prime}\right)$, one can get

$$
\left\{\begin{array}{l}
\mathcal{N}\left[\bar{F}\left(w, \chi(w), \chi^{\prime}(w), \gamma\right)\right]=\left[\frac{s^{2}}{u^{2}} \widehat{R}(s, u, \gamma) \ominus \frac{s}{u^{2}} \bar{\chi}_{0}(\gamma)\right] \ominus \frac{1}{u} \underline{\chi}_{0}^{\prime}(\gamma), \\
\mathcal{N}\left[\underline{F}\left(w, \chi(w), \chi^{\prime}(w), \gamma\right)\right]=\left[\frac{s^{2}}{u^{2}} \widehat{R}(s, u, \gamma) \ominus \frac{s}{u^{2}} \underline{\chi}_{0}(\gamma)\right] \ominus \frac{1}{u} \bar{\chi}_{0}^{\prime}(\gamma) .
\end{array}\right.
$$

Assume that by solving Equation (70), one can obtain the solution as follows: 


$$
\left\{\begin{array}{l}
\underline{\widehat{R}}(s, u, \gamma)=R_{3}(s, u, \gamma), \\
\widehat{\widehat{R}}(s, u, \gamma)=G_{3}(s, u, \gamma) .
\end{array}\right.
$$

Taking inverse fuzzy natural transform of Equation (71), one can find

$$
\left\{\begin{array}{l}
\underline{\chi}(w, \gamma)=\mathscr{N}^{-1}\left\{R_{3}(s, u, \gamma)\right\}, \\
\bar{\chi}(w, \gamma)=\mathscr{N}^{-1}\left\{G_{3}(s, u, \gamma)\right\} .
\end{array}\right.
$$

Case IV. Let $\chi(w)$ and $\chi^{\prime}(w)$ are (ii)-differentiable, then using Theorem 21, in (60), one can get

$$
\left\{\begin{array}{l}
\mathcal{N}\left[\bar{F}\left(w, \chi(w), \chi^{\prime}(w), \gamma\right)\right]=\left[\left(-\frac{s}{u^{2}} \bar{\chi}(0, \gamma)\right) \ominus\left(-\frac{s^{2}}{u^{2}} \widehat{\widehat{R}}(s, u, \gamma)\right)\right] \ominus\left(-\frac{1}{u} \bar{\chi}^{\prime}(0, \gamma)\right), \\
\mathcal{N}\left[\underline{F}\left(w, \chi(w), \chi^{\prime}(w), \gamma\right)\right]=\left[\left(-\frac{s}{u^{2}} \underline{\chi}(0, \gamma)\right) \ominus\left(-\frac{s^{2}}{u^{2}} \underline{\widehat{R}}(s, u, \gamma)\right)\right] \ominus\left(-\frac{1}{u} \underline{\chi}^{\prime}(0, \gamma)\right) .
\end{array}\right.
$$

Using initial condition in (73), $\chi\left(w_{0}\right)=\chi_{0}=\left(\bar{\chi}_{0}, \underline{\chi}_{0}\right)$ and $\chi^{\prime}\left(w_{0}\right)=\chi_{0}^{\prime}=\left(\bar{\chi}_{0}^{\prime}, \underline{\chi}_{0}^{\prime}\right)$, one can get

$$
\left\{\begin{array}{l}
\mathcal{N}\left[\bar{F}\left(w, \chi(w), \chi^{\prime}(w), \gamma\right)\right]=\left[\frac{s^{2}}{u^{2}} \widehat{R}(s, u, \gamma) \ominus \frac{s}{u^{2}} \bar{\chi}_{0}(\gamma)\right] \ominus \frac{1}{u} \bar{\chi}_{0}^{\prime}(\gamma), \\
\mathcal{N}\left[\underline{F}\left(w, \chi(w), \chi^{\prime}(w), \gamma\right)\right]=\left[\frac{s^{2}}{u^{2}} \underline{\widehat{R}}(s, u, \gamma) \ominus \frac{s}{u^{2}} \underline{\chi}_{0}(\gamma)\right] \ominus \frac{1}{u} \underline{\chi}_{0}^{\prime}(\gamma) .
\end{array}\right.
$$

Assume that by solving Equation (74), one can obtain the solution as follows:

$$
\left\{\begin{array}{l}
\underline{\widehat{R}}(s, u, \gamma)=R_{4}(s, u, \gamma), \\
\widehat{\bar{R}}(s, u, \gamma)=G_{4}(s, u, \gamma) .
\end{array}\right.
$$

Taking inverse fuzzy natural transform of Equation (75), one can find

$$
\left\{\begin{array}{l}
\underline{\chi}(w, \gamma)=\mathscr{N}^{-1}\left\{R_{4}(s, u, \gamma)\right\} \\
\bar{\chi}(w, \gamma)=\mathscr{N}^{-1}\left\{G_{4}(s, u, \gamma)\right\}
\end{array}\right.
$$

\section{Examples}

Here, we discuss the numerical problem.

$$
\left\{\begin{array}{l}
\chi^{\prime \prime}(w)=p(w) \chi(w)+q(w) \chi^{\prime}(w)+c(w) \\
\chi\left(w_{0}\right)=\chi_{0}=\left(\underline{\chi}_{0}, \bar{\chi}_{0}\right) \\
\chi^{\prime}\left(w_{0}\right) \chi_{0}^{\prime}=\left(\underline{\chi}_{0}^{\prime}, \bar{\chi}_{0}^{\prime}\right)
\end{array}\right.
$$

where $p, q: Y \rightarrow R^{+}$be continuous functions such that for nonnegative $L,|p(w)| \leq L,|q(w)| \leq L-1$, and $c: Y \rightarrow \widehat{F}_{R}$ be continuous function for all $w \in Y$, while $Y=[0,3 / L]$. If the functions $\chi: Y \rightarrow \widehat{F}_{R}$, the following conditions for some nonnegative $\varepsilon$ hold:

$$
\begin{aligned}
& d_{H}\left(D \chi(w), \chi^{\prime}(w)\right)+d_{H} \\
& \quad \cdot\left(D \chi^{\prime}(w), p(w) \chi(w)+q(w) \chi^{\prime}(w)+c(w)\right) \leq \varepsilon
\end{aligned}
$$

Assume that, on setting $F\left(w, \chi(w), \chi^{\prime}(w)\right)=p(w) \chi(w)$ $+q(w) \chi^{\prime}(w)+w(w)$, it is easy to obtain condition (i), as follows

$$
\begin{aligned}
d_{H}( & F\left(w, \chi_{1}, \chi_{1}^{\prime}\right), F\left(w, \chi_{2}, \chi_{2}^{\prime}\right) \\
= & d_{H}\left(p(w) \chi_{1}(w)+q(w) \chi_{1}^{\prime}(w)+c(w), p(w) \chi_{2}(w)\right. \\
& \left.+q(w) \chi_{2}^{\prime}(w)+c(w)\right) \leq d_{H}\left(p(w) \chi_{1}(w), p(w) \chi_{2}(w)\right) \\
& +d_{H}\left(q(w) \chi_{1}^{\prime}(w), q(w) \chi_{2}^{\prime}(w)\right)=|p(w)| d_{H}\left(\chi_{1}(w), \chi_{2}(w)\right) \\
& +|q(w)| d_{H}\left(\chi_{1}^{\prime}(w), \chi_{2}^{\prime}(w)\right) \leq L d_{H}\left(\chi_{1}(w), \chi_{2}(w)\right) \\
& +(L-1) d_{H}\left(\chi_{1}^{\prime}(w), \chi_{2}^{\prime}(w)\right), \quad \text { for all } w \in Y
\end{aligned}
$$

Then, there exist unique solution of the problem (77), $\widehat{u}, \widehat{v}: Y \rightarrow \widehat{F}_{R}$, defined by

$$
\left\{\begin{array}{l}
\widehat{\chi}=\chi_{0}+\int_{3 / 2 L}^{w} \chi^{\prime}(s) d s \\
\widehat{\chi}^{\prime}=\chi_{0}^{\prime}+\int_{3 / 2 L}^{w} F\left(s, \chi(s), \chi^{\prime}(s)\right) d s
\end{array}\right.
$$

$$
\begin{aligned}
d_{H}(\chi, \widehat{\chi}) & \leq(1+L) \frac{3}{2 L} \varepsilon, \\
d_{H}\left(\chi^{\prime}, \widehat{\chi}^{\prime}\right) & \leq(1+L) \frac{3}{2 L} \varepsilon, \quad \text { for all, } w \in Y .
\end{aligned}
$$

This shows that the fuzzy solution of differential equations, (77), is Hyers-Ulam stable on $Y$.

When $p(w)=p$ and $q(w)=q$, then $R_{1}(s, u, \gamma)$ and $G_{1}(s$, $u, \gamma)$ of system (60) can be calculated as

Case 1. When $p, q \geq 0$, then system (61) is equivalent to

$$
\left\{\begin{array}{l}
\frac{s^{2}}{u^{2}} \underline{\underline{R}}(s, u, \gamma) \ominus \frac{s}{u^{2}} \underline{\chi}(0, \gamma) \ominus \frac{1}{u} \underline{\chi}^{\prime}(0, \gamma)=\left(p+q \frac{s}{u}\right) \hat{\underline{R}}(s, u, \gamma) \ominus q \frac{1}{u} \underline{\chi}^{\prime}(0, \gamma)+N(\underline{c}(w)), \\
\frac{s^{2}}{u^{2}} \widehat{\bar{R}}(s, u, \gamma) \ominus \frac{s}{u^{2}} \bar{\chi}(0, \gamma) \ominus \frac{1}{u} \bar{\chi}^{\prime}(0, \gamma)=\left(p+q \frac{s}{u}\right) \widehat{\bar{R}}(s, u, \gamma) \ominus q \frac{1}{u} \bar{\chi}^{\prime}(0, \gamma)+N(\bar{c}(w)) .
\end{array}\right.
$$

After some process, one can get

$$
\left\{\begin{array}{l}
R_{1}(s, u, \gamma)=\underline{\widehat{R}}(s, u, \gamma)=\frac{\left(s / u^{2}-q / u\right) \underline{\chi}(0, \gamma)+(1 / u) \underline{\chi}^{\prime}(0, \gamma)+N(\underline{c}(w))}{s^{2} / u^{2}-q s / u-p} \\
G_{1}(s, u, \gamma)=\widehat{\widehat{R}}(s, u, \gamma)=\frac{\left(s / u^{2}-q / u\right) \bar{\chi}(0, \gamma)+(1 / u) \bar{\chi}^{\prime}(0, \gamma)+N(\bar{c}(w))}{s^{2} / u^{2}-q s / u-p}
\end{array}\right.
$$


Case 2. When $p \geq 0$ and $q<0$, then system (65) is equivalent to the following:

$$
\left\{\begin{array}{l}
\frac{s^{2}}{u^{2}} \underline{\widehat{R}}(s, u, \gamma) \ominus \frac{s}{u^{2}} \underline{\chi}(0, \gamma) \ominus \frac{1}{u} \underline{\chi}^{\prime}(0, \gamma)=p \underline{\widehat{R}}(s, u, \gamma)+q \frac{s}{u} \widehat{\bar{R}}(s, u, \gamma) \ominus \frac{q}{u} \bar{\chi}^{\prime}(0, \gamma)+N(\underline{c}(w)), \\
\frac{s^{2}}{u^{2}} \widehat{\bar{R}}(s, u, \gamma) \ominus \frac{s}{u^{2}} \bar{\chi}(0, \gamma) \ominus \frac{1}{u} \bar{\chi}^{\prime}(0, \gamma)=p \widehat{\bar{R}}(s, u, \gamma)+q \frac{s}{u} \widehat{\widehat{R}}(s, u, \gamma) \ominus \frac{q}{u} \underline{\chi}^{\prime}(0, \gamma)+N(\bar{c}(w)) .
\end{array}\right.
$$

Let us denote

$$
\left\{\begin{array}{l}
f(s, u, \gamma)=\frac{s}{u^{2}} \underline{\chi}(0, \gamma)+\frac{1}{u} \underline{\chi^{\prime}}(0, \gamma)-\frac{q}{u} \bar{\chi}^{\prime}(0, \gamma)+N(\underline{c}(w)), \\
g(s, u, \gamma)=\frac{s}{u^{2}} \bar{\chi}(0, \gamma)+\frac{1}{u} \bar{\chi}^{\prime}(0, \gamma)-\frac{q}{u} \underline{\chi}^{\prime}(0, \gamma)+N(\bar{c}(w)) .
\end{array}\right.
$$

Solving the above system, one can get

$$
\left\{\begin{array}{l}
R_{1}(s, u, \gamma)=\underline{\widehat{R}}(s, u, \gamma)=\frac{\left(s^{2} / u^{2}-p\right) f(s, u, \gamma)+(q s / u) g(s, u, \gamma)}{\left(s^{2} / u^{2}-p\right)^{2}+(q s / u)^{2}}, \\
G_{1}(s, u, \gamma)=\widehat{R}(s, u, \gamma)=\frac{\left(s^{2} / u^{2}-p\right) g(s, u, \gamma)-(q s / u) f(s, u, \gamma)}{\left(s^{2} / u^{2}-p\right)^{2}+(q s / u)^{2}} .
\end{array}\right.
$$

Case 3. When $p<0$ and $q \geq 0$, then system (69) is equivalent to the following system:

$$
\left\{\begin{array}{l}
\frac{s^{2}}{u^{2}} \underline{\widehat{R}}(s, u, \gamma) \ominus \frac{s}{u^{2}} \underline{\chi}(0, \gamma) \ominus \frac{1}{u} \underline{\chi}^{\prime}(0, \gamma)=p \widehat{\widehat{R}}(s, u, \gamma)+q \frac{s}{u} \underline{\widehat{R}}(s, u, \gamma) \ominus \frac{q}{u} \underline{\chi}^{\prime}(0, \gamma)+N(\underline{c}(w)), \\
\frac{s^{2}}{u^{2}} \widehat{\widehat{R}}(s, u, \gamma) \ominus \frac{s}{u^{2}} \bar{\chi}(0, \gamma) \ominus \frac{1}{u} \bar{\chi}^{\prime}(0, \gamma)=p \underline{\widehat{R}}(s, u, \gamma)+q \frac{s}{u} \widehat{\bar{R}}(s, u, \gamma) \ominus \frac{q}{u} \bar{\chi}^{\prime}(0, \gamma)+N(\bar{c}(w)) .
\end{array}\right.
$$

Let us denote

$$
\left\{\begin{array}{l}
B(s, u, \gamma)=\frac{s}{u^{2}} \underline{\chi}(0, \gamma)+\frac{1}{u} \underline{\chi}^{\prime}(0, \gamma)-\frac{q}{u} \underline{\chi}^{\prime}(0, \gamma)+N(\underline{c}(w)), \\
C(s, u, \gamma)=\frac{s}{u^{2}} \bar{\chi}(0, \gamma)+\frac{1}{u} \bar{\chi}^{\prime}(0, \gamma)-\frac{q}{u} \bar{\chi}^{\prime}(0, \gamma)+N(\bar{c}(w)) .
\end{array}\right.
$$

Solving the above system, one can get

$$
\left\{\begin{array}{l}
R_{1}(s, u, \gamma)=\underline{\widehat{R}}(s, u, \gamma)=\frac{\left(s^{2} / u^{2}-q s / u\right) B(s, u, \gamma)+p C(s, u, \gamma)}{\left(s^{2} / u^{2}-q s / u\right)^{2}+(p)^{2}}, \\
G_{1}(s, u, \gamma)=\widehat{\widehat{R}}(s, u, \gamma)=\frac{\left(s^{2} / u^{2}-q s / u\right) C(s, u, \gamma)+p B(s, u, \gamma)}{\left(s^{2} / u^{2}-q s / u\right)^{2}+(p)^{2}} .
\end{array}\right.
$$

Case 4. When $p<0$ and $q<0$, then system (73) is equivalent to the following:

$$
\left\{\begin{array}{l}
\frac{s^{2}}{u^{2}} \underline{\widehat{R}}(s, u, \gamma) \ominus \frac{s}{u^{2}} \underline{\chi}(0, \gamma) \ominus \frac{1}{u} \underline{\chi}^{\prime}(0, \gamma)=p \widehat{\widehat{R}}(s, u, \gamma)+q \frac{s}{u} \widehat{\bar{R}}(s, u, \gamma) \ominus \frac{q}{u} \bar{\chi}^{\prime}(0, \gamma)+N(\underline{c}(w)), \\
\frac{s^{2}}{u^{2}} \widehat{\bar{R}}(s, u, \gamma) \ominus \frac{s}{u^{2}} \bar{\chi}(0, \gamma) \ominus \frac{1}{u} \bar{\chi}^{\prime}(0, \gamma)=p \underline{\widehat{R}}(s, u, \gamma)+q \frac{s}{u} \underline{\widehat{R}}(s, u, \gamma) \ominus \frac{q}{u} \underline{\chi}^{\prime}(0, \gamma)+N(\bar{c}(w)) .
\end{array}\right.
$$



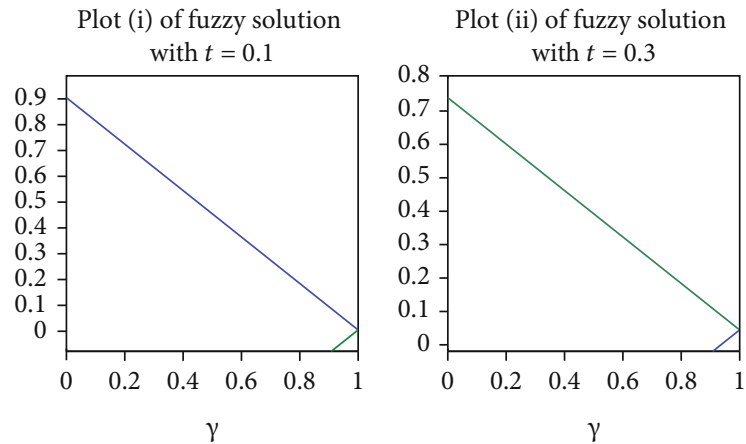

Figure 1: Present fuzzy solutions of Example 1 for different values of $\gamma$. The subplot (i) is 2D plot of lower and upper solutions with $t=0.1$, and subplot (ii) is $2 \mathrm{D}$ plot of lower and upper solutions with $t=0.3$.

For the remaining system, we can find the same processes. Solving the above system, one can get

$$
\left\{\begin{array}{l}
R_{1}(s, u, \gamma)=\underline{\widehat{R}}(s, u, \gamma)=\frac{\left(s^{2} / u^{2}\right) \mathrm{f}(s, u, \gamma)+(p+q s / u) g(s, u, \gamma)}{\left(s^{2} / u^{2}\right)^{2}+(p+q s / u)^{2}} \\
G_{1}(s, u, \gamma)=\widehat{R}(s, u, \gamma)=\frac{\left(s^{2} / u^{2}\right) g(s, u, \gamma)+(p+(q s / u)) f(s, u, \gamma)}{\left(s^{2} / u^{2}\right)^{2}+(p+q s / u)^{2}} .
\end{array}\right.
$$

Plot (ii) of fuzzy solution with $t=0.5$
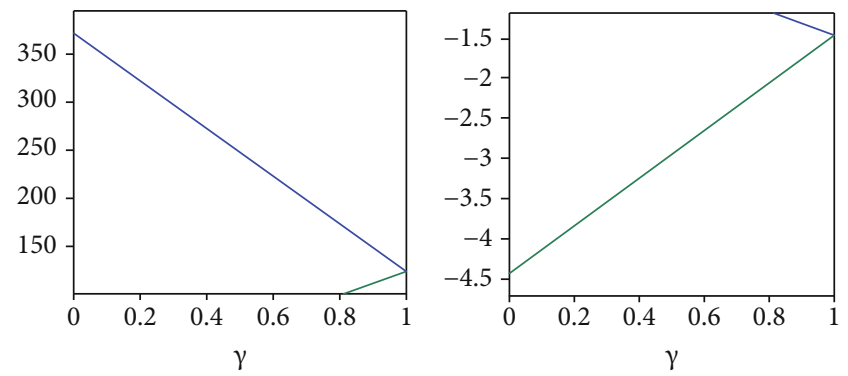

Figure 2: Present fuzzy solutions of Example 2 for different values of $\gamma$. The subplot (i) is 2D plot of lower and upper solutions with $t=5$, and subplot (ii) is $2 \mathrm{D}$ plot of lower and upper solutions with $t=0.5$.

This differential equation is equivalent to Case 3 of problem (77); therefore, the solution exists.

$\left\{\begin{array}{l}\frac{s^{2}}{u^{2}} \underline{\widehat{R}}(s, u, \gamma) \ominus \frac{s}{u^{2}}(\gamma-1) \ominus \frac{1}{u}(\gamma-1)=-\widehat{\widehat{R}}(s, u, \gamma)+N(\underline{w}(t)), \\ \frac{s^{2}}{u^{2}} \widehat{\widehat{R}}(s, u, \gamma) \ominus \frac{s}{u^{2}}(1-\gamma) \ominus \frac{1}{u}(1-\gamma)=-\underline{\widehat{R}}(s, u, \gamma)+N(\bar{w}(t)) .\end{array}\right.$

Example 1. Now, we discuss the following system:

$$
\left\{\begin{array}{l}
\chi^{\prime \prime}(t)=-\chi(t)+w_{0}, \quad w_{0}=(\gamma, 2-\gamma) \\
\chi(0, \gamma)=(\gamma-1,1-\gamma) \\
\chi^{\prime}(0, \gamma)=(\gamma-1,1-\gamma)
\end{array}\right.
$$

$$
\left\{\begin{array}{l}
R_{1}(s, u, \gamma)=\underline{\widehat{R}}(s, u, \gamma)=\gamma\left(\frac{1}{2(s+u)}-\frac{1}{2(s-u)}+\frac{1}{s}\right)+\frac{1}{2(s-u)}-\frac{1}{2(s+u)}-\frac{s}{s^{2}+\mathrm{u}^{2}}, \\
G_{1}(s, u, \gamma)=\widehat{\vec{R}}(s, u, \gamma)=\gamma\left(\frac{1}{2(s-u)}-\frac{1}{2(s+u)}-\frac{1}{s}\right)+\frac{2}{s}+\frac{1}{2(s+u)}-\frac{1}{2(s-u)}-\frac{s}{s^{2}+u^{2}} .
\end{array}\right.
$$

Now, applying inverse natural transform, we deduce

$$
\left\{\begin{array}{l}
\underline{\chi}(t, \gamma)=\gamma(1-\sinh (t))+\sinh (t)-\cos (t), \\
\bar{\chi}(t, \gamma)=(2-\gamma)(1-\sinh (t))+\sinh (t)-\cos (t) .
\end{array}\right.
$$

This solution is acceptable for all $t \in(0, \ln (1+\sqrt{ } 2))$, see [30] (Figure 1).
Example 2. Here, we consider the following system:

$$
\left\{\begin{array}{l}
\chi^{\prime \prime}(t)=\chi(t)+t+1 \\
\chi(0, \gamma)=(\gamma-2,1-2 \gamma) \\
\chi^{\prime}(0, \gamma)=(\gamma-2,1-2 \gamma)
\end{array}\right.
$$

This differential equation is equivalent to Case 1 of problem (77); therefore, the solution exists. 
$\left\{\begin{array}{l}\frac{s^{2}}{u^{2}} \underline{\widehat{R}}(s, u, \gamma) \ominus \frac{s}{u^{2}}(\gamma-2) \ominus \frac{1}{u}(\gamma-2)=-\widehat{\bar{R}}(s, u, \gamma)+\frac{u}{s^{2}}+\frac{1}{s}, \\ \frac{s^{2}}{u^{2}} \widehat{\widehat{R}}(s, u, \gamma) \ominus \frac{s}{u^{2}}(1-\gamma) \ominus \frac{1}{u}(1-\gamma)=-\underline{\widehat{R}}(s, u, \gamma)+\frac{u}{s^{2}}+\frac{1}{s} .\end{array}\right.$

Solving Equation (97), one can write

$$
\left\{\begin{array}{l}
R_{1}(s, u, \gamma)=\underline{\widehat{R}}(s, u, \gamma)=\frac{\gamma}{s-u}-\frac{2}{s-u}+\frac{1}{s^{2}(s-u)}+\frac{1}{s^{3}(s-u)}, \\
G_{1}(s, u, \gamma)=\widehat{\bar{R}}(s, u, \gamma)=\frac{1}{s-u}-\frac{2 \gamma}{s-u}+\frac{1}{s^{2}(s-u)}+\frac{1}{s^{3}(s-u)} .
\end{array}\right.
$$

Now, applying inverse natural transform, we deduce

$$
\left\{\begin{array}{l}
\underline{\chi}(t, \gamma)=\gamma \exp (t)-\frac{t^{2}}{2}-2 t-2 \\
\bar{\chi}(t, \gamma)=(3-2 \gamma) \exp (t)-\frac{t^{2}}{2}-2 t-2
\end{array}\right.
$$

This solution is acceptable for all $t \in R$ (real numbers) (Figure 2).

\section{Conclusion and Future Direction}

In this work, we study the existence, uniqueness, and HyersUlam stability of second-order FDEs. We discuss the natural transform of second-order fuzzy differential equations for different cases of fuzzy differentiability. The natural transform converts to Laplace transform by taking parameter $p$ $=1$ and Sumudu transform by taking parameter $s=1$. Therefore, we use natural transform to solve the proposed FDEs. In the future, the natural transform method will be used to solve fuzzy problems due to significant duality with Laplace and Sumudu transforms.

\section{Data Availability}

No date were used to support this study.

\section{Conflicts of Interest}

The authors declare that they have no competing interest regarding this manuscript.

\section{Authors' Contributions}

All authors contributed equally to the writing of this manuscript. All authors read and approve the final version.

\section{Acknowledgments}

The authors would like to extend their sincere appreciation to the Deanship of Scientific Research, King Saud University for its funding through the Research Unit of Common First Year Deanship.

\section{References}

[1] L. A. Zadeh, "Fuzzy sets," Information and Control, vol. 8, no. 3, pp. 338-353, 1965.

[2] S. S. Chang and L. A. Zadeh, "On fuzzy mapping and control," in Fuzzy sets, fuzzy logic, and fuzzy systems, L. A. Zadeh, Ed., World Scientific, 1996.

[3] D. Dubois and H. Prade, "Towards fuzzy differential calculus part 3: differentiation," Fuzzy Sets and Systems, vol. 8, no. 3, pp. 225-233, 1982.

[4] R. Goetschel Jr. and W. Voxman, "Elementary fuzzy calculus," Fuzzy Sets and Systems, vol. 18, no. 1, pp. 31-43, 1986.

[5] O. Kaleva, "Fuzzy differential equations," Fuzzy Sets and Systems, vol. 24, no. 3, pp. 301-317, 1987.

[6] A. Khastan and R. Rodríguez-López, "An existence and uniqueness result for fuzzy Goursat partial differential equation," Fuzzy Sets and Systems, vol. 375, pp. 141-160, 2019.

[7] H. Khan, F. Jarad, T. Abdeljawad, and A. Khan, "A singular ABC-fractional differential equation with $p$-Laplacian operator," Chaos, Solitons \& Fractals, vol. 129, pp. 56-61, 2019.

[8] B. Liu and Y.-K. Liu, "Expected value of fuzzy variable and fuzzy expected value models," IEEE Transactions on Fuzzy Systems, vol. 10, no. 4, pp. 445-450, 2002.

[9] W. Fei, "Uniqueness of solutions to fuzzy differential equations driven by Lius process with nonLipschitz coefficients," in 2009 Sixth International Conference on Fuzzy Systems and Knowledge Discovery, pp. 565-569, Tianjin, China, August 2009.

[10] X. Chen and X. Qin, "A new existence and uniqueness theorem for fuzzy differential equations," International Journal of Fuzzy Systems, vol. 13, no. 2, pp. 148-151, 2013.

[11] A. Harir, S. Melliani, and L. S. Chadli, "Existence and uniqueness of a fuzzy solution for some fuzzy neutral partial differential equation with nonlocal condition," International Journal of Mathematics Trends and Technology, vol. 65, no. 2, article 102107, 2019.

[12] J. Ahmad and M. Mushtaq, "Exact solution of linear and nonlinear Goursat problems," Universal Journal of Computational Mathematics, vol. 3, article 1417, 2015.

[13] S. M. Ulam, A Collection of the Mathematical Problems, Interscience, New York, NY, USA, 1940.

[14] D. H. Hyers, "On the stability of the linear functional equation," Proceedings of the National Academy of Sciences of the United States of America, vol. 27, no. 4, pp. 222-224, 1941.

[15] M. Oblaza, "Hyers stability of the linear differential equation," Rocznik Nauk.-Dydakt. PraceMatematyczne, vol. 13, pp. 259270, 1993.

[16] S.-M. Jung and J. Brzdek, "Hyers-Ulam stability of the delay equation $y(t)=\lambda(t-\tau)$," Abstract and Applied Analysis, vol. 2010, Article ID 372176, 10 pages, 2010.

[17] A. Zada, W. Ali, and C. Park, "Ulam's type stability of higher order nonlinear delay differential equations via integral inequality of Gronwall-Bellman-Bihari's type," Applied Mathematics and Computation, vol. 350, pp. 60-65, 2019.

[18] I. Ullah, S. Ahmad, Q. Al-Mdallal, Z. A. Khan, H. Khan, and A. Khan, "Stability analysis of a dynamical model of tuberculosis with incomplete treatment," Advances in Difference Equations, vol. 2020, no. 1, 2020.

[19] A. Shah, R. A. Khan, A. Khan, H. Khan, and J. F. Gmez-Aguilar, "Investigation of a system of nonlinear fractional order hybrid differential equations under usual boundary conditions 
for existence of solution," Mathematical Methods in the Applied Sciences, vol. 44, no. 2, pp. 1628-1638, 2021.

[20] Y. Shen, "On the Ulam stability of first order linear fuzzy differential equations under generalized differentiability," Fuzzy Sets and Systems, vol. 280, pp. 27-57, 2015.

[21] Z. H. Khan and W. A. Khan, "N-transform properties and application," NUST Journal of Engineering Sciences, vol. 1, pp. 127-133, 2008.

[22] K. Shah, M. Junaid, and N. Ali, "Extraction of Laplace, Sumudu, Fourier and Mellin transform from the natural transform," Journal of Applied Environmental and Biological Sciences, vol. 5, no. 9, pp. 1-8, 2015.

[23] D. Loonker and P. K. Banerii, "Natural transform and solution of integral equations for distribution spaces," American Journal of Mathematics and Sciences, vol. 3, no. 1, pp. 2250-3102, 2014.

[24] B. Bede, Mathematics of Fuzzy Sets and Fuzzy Logic, Springer, London, 2013.

[25] M. Friedman, M. Ma, and A. Kandel, "Numerical solutions of fuzzy differential and integral equations," Fuzzy Sets and Systems, vol. 106, no. 1, pp. 35-48, 1999.

[26] P. Diamond and P. Kloeden, Metric Spaces of Fuzzy Sets, World Scientific, Singapore, 1994.

[27] B. Bede, I. J. Rudas, and A. L. Bencsik, "First order linear fuzzy differential equations under generalized differentiability," Information Sciences, vol. 177, no. 7, pp. 1648-1662, 2007.

[28] O. Kaleva, "A note on fuzzy differential equations," Nonlinear Analysis, vol. 64, no. 5, pp. 895-900, 2006.

[29] Y. Chalco-Cano and H. Román-Flores, "On new solutions of fuzzy differential equations," Chaos Solitons Fractals, vol. 38, no. 1, pp. 112-119, 2008.

[30] T. Allahviranloo, S. Abbasbandy, S. Salahshour, and A. Hakimzadeh, "A new method for solving fuzzy linear differential equations,” Computing, vol. 92, no. 2, pp. 181-197, 2011. 